\title{
A simulation study on the effect of ionic currents on transmission from cones to retinal OFF type cone bipolar cells
}

Akito Ishihara

Department of Mechanical and Systems Engineering, School of Engineering, Chukyo University, Nagoya, Japan

Keywords: glutamate receptor, ionic current light response, retinal OFF type bipolar cell

\section{Background and purpose}

The retinal cone bipolar cells are interneurons which receive inputs from cone photoreceptors and send outputs to retinal ganglion cells. Several subtypes of bipolar cells have been identified by morphology and electrophysiology in the mammalian retina, which convey distinct visual information to higher order neurons in parallel. ${ }^{1}$ The neural circuit in the retina not only converts light information to neural information, but also performs visual information preprocessing that has not yet been fully understood. Recently, it has been revealed that the neural circuits in retinas of higher vertebrates, such as mammals and primates, have various biophysical properties arising from being composed of ionic channels, ionic pumps, and neurotransmitter receptors. Analysis using a mathematical model based on their ionic mechanisms is essential to understand the visual information processing in the retinal neural circuit of the higher vertebrates.

The cones and the bipolar cells respond to continuous variation of light with a graded potential, in an analog manner. Especially, glutamate is continuously released from a cone synapse in the dark and is decreased by hyperpolarization of the

Correspondence: Akito Ishihara, 101-2 Yagoto-honmachi, Showa-ku, Nagoya, 466-8666 Japan. E-mail: aishi@sist.chukyo-u.ac.jp 
cone that receives the light stimulus. The alpha-amino-3-hydroxy-5-methyl-4-isoxazolepropionic acid (AMPA) and kainate type ionotropic glutamate receptors (iGluRs) of the OFF type bipolar cells (OFF-BCs) exhibit partial or nearly complete desensitization in the sustained presence of glutamate. ${ }^{2-4}$ In the dark, glutamate concentration in the synaptic cleft of the cone pedicle rises to $0.1-0.5 \mathrm{mM} .{ }^{5,6}$ The baseline glutamate concentration depends on a sustained hyperpolarization of the cone by light. Thus, for understanding the working of the OFF-BCs, it is important to elucidate the mechanisms of synaptic transmission from cones to OFF-BCs via iGluRs, which undergo desensitization in the various background light conditions. Furthermore, there are various kinds of ionic channels in OFF-BCs that mediate membrane potential responses. ${ }^{7-9}$ It is considered that information transmitted from cones to OFF-BCs is modulated by the intrinsic ionic currents. We analyzed how ionic currents of OFF-BCs contribute to the transmission of light responses by developing a mathematical model.

\section{Methods}

To analyze the effect of ionic currents on transmission properties from cones to OFF-BCs, we constructed a mathematical model based on their ionic currents, as shown in the left panel in Figure 1. The OFF-BC model consists of an iGluR-mediated current and two voltage-dependent ionic currents in the soma, a delayed rectifying potassium current $\left(i_{K v}(\mathrm{t})\right)$, and an inward rectifying current $\left(i_{h}(\mathrm{t})\right)$. The models of these ionic currents have already been proposed in our previous research. ${ }^{10,11}$ Briefly, the parameters of $i_{k v}(\mathrm{t})$ and $i_{h}(\mathrm{t})$ which are described by the Hodgkin-Hux-
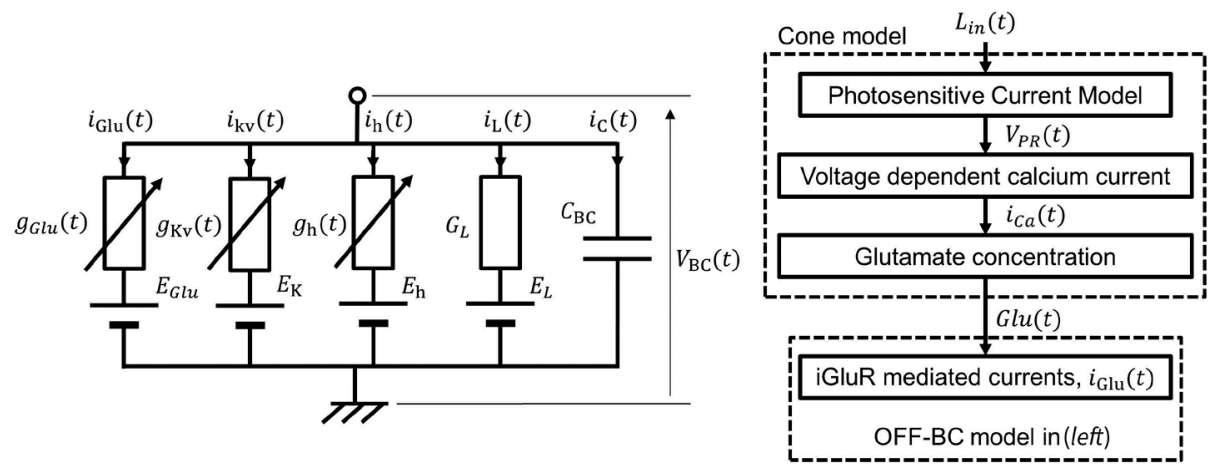

Fig 1. (Left) OFF-BCs were modeled by a parallel conductance model that introduced an iGluR-mediated current $\left(i_{G l u}(t)\right)$, a delayed rectifying potassium current $\left(i_{K v}(t)\right)$, an inward rectifying current $\left(i_{h}(t)\right)$, and a leakage current $\left(i_{L}(t)\right)$. (Right) Glutamate concentration $(G l u(t))$ in the postsynaptic site was modulated by voltage-dependent calcium current $\left(i_{c a}(t)\right)$, which was inactivated by membrane hyperpolarization of the cone $\left(V_{P R}(t)\right)$. 
ley type equation, were re-estimated by using electrophysiological data from the mammalian retina. ${ }^{7,8}$

The simulations of light responses of OFF-BCs were calculated by a network model which was constructed by adding the cone model ${ }^{12}$ to the OFF-BC model. The membrane potential of the cone model $\left(V_{P R}(t)\right)$ was hyperpolarized to light stimuli by a photo-sensitive current. After the membrane potential change at the inner segment propagates to the cone pedicle, the hyperpolarization reduces glutamate release due to inactivation of the voltage dependent calcium current $\left(i_{c a}\right)$. The response of glutamate concentration $(\mathrm{Glu}(\mathrm{t}))$ at the postsynaptic site was modeled simply with the $i_{C a}$ system. ${ }^{13}$

Some model parameters were not able to be estimated due to lack of physiological data. We analyzed the effect of the model parameters on synaptic transmission properties and light responses of OFF-BCs. Numerical solutions and computer simulations were obtained using the developed model and run using Matlab (Mathworks, Natick, MA, USA).

\section{Results}

We computationally analyzed the effect of the kinetics of iGluR-mediated currents and somatic voltage-dependent currents on responses of OFF-BCs to sinusoidal glutamate inputs. The left panel in Figure 2 shows the simulated responses of the OFF-BC model (Fig. 2, bottom left) to sinusoidal glutamate inputs (Fig. 2, top left). The baseline of $V_{B C}$ was hyperpolarized and the amplitude of $V_{B C}$ response was increased when glutamate concentration was decreased (Fig. 2, blue line in left panel). The right panel in Figure 2 shows the relationship of the peak-to-peak amplitude of the $V_{B C}\left(V_{p p}\right)$ with the temporal frequency of inputs. The responses under high glutamate concentration showed a band-pass filtering feature (Fig. 2, black open circles in right panel), whereas the frequency profile showed a low-pass filtering manner in the low baseline glutamate concentrations (Fig. 2, blue filled circles in right panel). Our analysis of the model responses (data not shown) showed that the upper cutoff frequency of the band-pass filtering responses depends on the rate of desensitization of iGluR and the membrane time constant, which is given by the membrane capacitance $\left(C_{B C}\right)$, the sum of the leakage conductance $\left(G_{L}\right)$, and other ionic conductance. The frequency characteristics also depend on $i_{k v}$ and $i_{h}$. $V_{p p}$ to low-frequency inputs were decreased with increased maximal conductance of $i_{K V}$ and $i_{h}$ (Fig. 2, open circles in right panel), when at least one of the $i_{K v}$ or $i_{h}$ was activated at $V_{B C}$. These results suggest that transmission of temporal low-frequency signals in OFF-BCs depends on the baseline glutamate concentrations in the postsynaptic site, which are mainly controlled by the mean light intensity. This also suggests that the components of OFF-BC responses are modified by ionic current rectification of the membrane. 


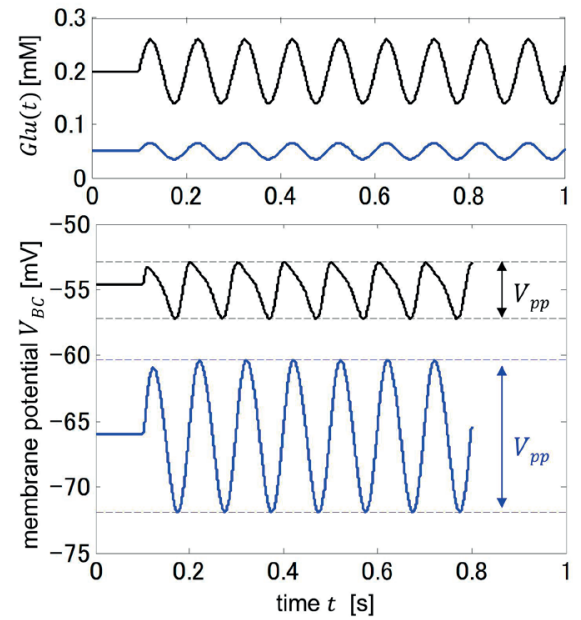

(a)

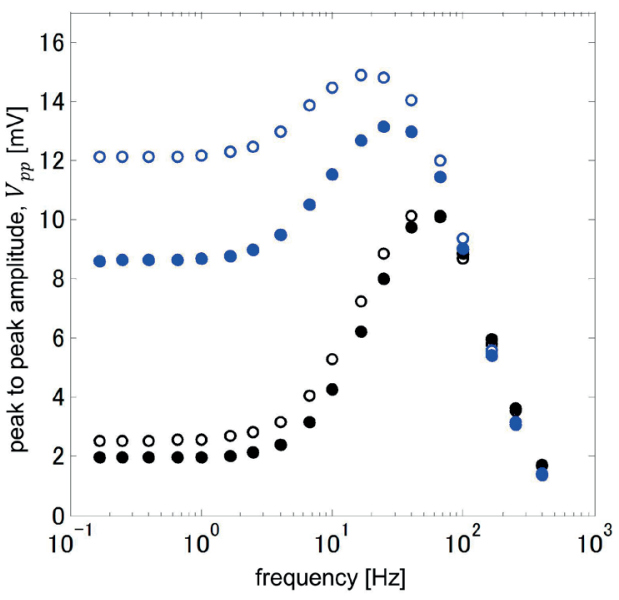

(b)

Fig 2. (Left) Time course of membrane potential responses of the OFF-BC model $\left(V_{B C}\right.$, bottom) to sinusoidal glutamate wave inputs (top) with frequency of $10 \mathrm{~Hz}$ and amplitude of $30 \%$ of the baseline value of $0.2 \mathrm{mM}$ (black line), $0.05 \mathrm{mM}$ (blue line). (Right) The peak-to-peak amplitude of $V_{B C}\left(V_{p p}\right.$, the arrows in (left)) were plotted versus the frequency of the sinusoidal stimulus under a baseline glutamate concentration of $0.2 \mathrm{mM}$ (black circles), $0.05 \mathrm{mM}$ (blue circles). Open circles indicate the frequency characteristics of the model without $i_{K v}$ and $i_{h}$; filled circles with $i_{K v}$ and $i_{h}$.

We next analyzed the effect of ionic mechanisms in OFF-BCs to light responses of the cells by using the network model of a cone and an OFF-BC. OFF-BC responses showed a stronger temporal band-pass filtering feature (Fig. 3, bottom left) than cone responses (Fig. 3, top left). In the cone, the frequency relationship of responses to mean intensity of $4000 \mathrm{td}$ (Fig. 3, black circles in left panel) were the same as that to a mean intensity of $10000 \mathrm{td}$ (Fig. 3, blue circles in left panel). In the OFF-BC, the aforementioned frequency relationship is different in both of the mean intensities (Fig. 3, bottom left). The right panel in Figure 3 shows the time courses of $V_{P R}(\mathrm{t}), \mathrm{Glu}(\mathrm{t})$ and $V_{B C}(\mathrm{t})$ with response to a mean intensity of $4000 \mathrm{td}$ (Fig. 3, black dashed lines in right panel) and 10000 td (Fig. 3, blue solid lines in right panel). The ratio (C, Fig. 3, in set in right panel) of peak-to-peak amplitudes of OFF-BC fluctuation response to a mean intensity of $10000 \mathrm{td}$ and $4000 \mathrm{td}$ was greater than 1 , although the ratio of amplitudes of $\mathrm{Glu}(\mathrm{t})$ response was nearly equal to 1 . The results suggest that the difference of the frequency characteristics of OFF-BC response was produced by the iGluR current and their somatic ionic currents.

OFF-BC responses were strongly attenuated by the light stimulus with a mean intensity of $1000 \mathrm{td}$, under which both the cones and rods act. In this dark condition, 

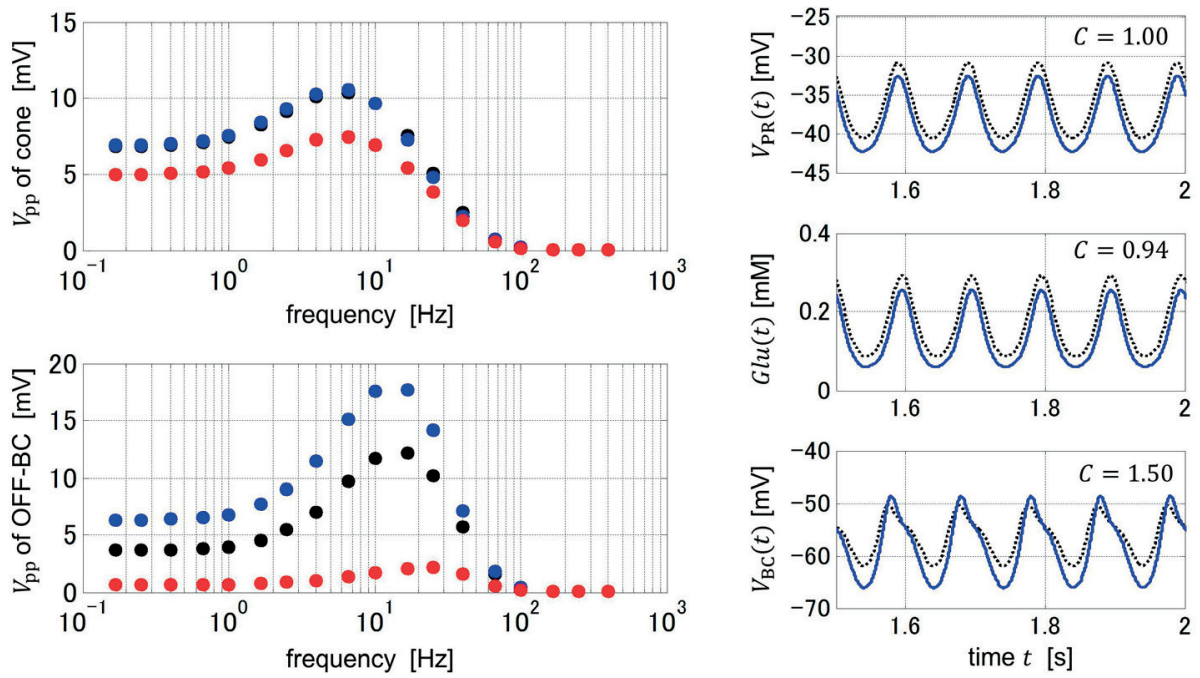

Fig 3. (Left) Relationship $V_{p p}$ of the cone model (top) and the OFF-BC model (bottom) with the frequency of sinusoidal light inputs with amplitude of $60 \%$ of mean intensities of 1000 td (red circles), $4000 \mathrm{td}$ (black circles), and $10000 \mathrm{td}$ (blue circles). (Right) Time course of the response of $V_{P R}(t)$ (top), Glu(t) (middle), and $V_{B C}(t)$ (bottom) to sinusoidal light stimulus with a frequency of $10 \mathrm{~Hz}$. The black dashed lines indicate responses under the mean intensity of $4000 \mathrm{td}$ and the blue solid lines indicate those under $10000 \mathrm{td}$. The values of $C$ (in set) express the ratio between the $V_{p p}$ of responses to the mean intensity $10000 \mathrm{td} / 4000 \mathrm{td}$.

the glutamate concentration in the postsynaptic site was high. Therefore, the OFF-BC responses were suppressed by desensitization of iGluRs and depolarization of mean membrane potential (Fig. 2, black line in left panel). Our simulation analysis using square pulse light waves demonstrated that the OFF-BC transmit temporal transient signals such as ON-OFF signals under the dark-illumination condition..$^{10,14}$

\section{Conclusions and future perspectives}

The results show that the temporal frequency responses of the OFF-BCs depend on the iGluR current and the somatic ionic currents. Moreover, its characteristics are modulated by mean illumination. These results suggest that the iGluR current acts to transmit low pass filtering signals under the day-light condition and it acts to attenuate temporal low-frequency signals under the dark condition. Furthermore, the results suggest that somatic ionic currents $i_{k v}$ and $i_{h}$ also attenuate low-frequency signals of high mean light instensity.

Our simulated results showed that the transmission from a cone to an OFF-BC adaptively change with mean light intensity in the mammalian retina. It has been 
reported that bipolar cells in the mammalian retina have voltage-gated sodium channels ${ }^{6,15}$ and some subtypes of the cells respond to light with a spike. ${ }^{13}$ Since the sodium channels are activated from a hyperpolarized potential to depolarization, it is possible that light responses under dark conditions are affected by current through the channels, if there are channels in OFF-BCs. Other ionic currents such as calcium-dependent currents have been reported in the synaptic terminal of bipolar cells. ${ }^{1}$ By modeling the bipolar cells with integration of these ionic mechanisms, we can help elucidate how these cells play a role in visual information processing.

The five types of OFF-BCs in the mammalian retina are characterized by the temporal response to glutamate application. ${ }^{3,4}$ It is expected that OFF signals of visual information could be processed in parallel by these five channels. ${ }^{4}$ With the improvement of our model based on the electrophysiological evidence, we expect that visual information processing in the OFF pathway of the higher vertebrate retina will be clarified in detail, especially with respect as to why the five channels are needed.

\section{Acknowledgements}

This research was partially supported by the Ministry of Education, Culture, Sports, Science and Technology (Japan) through a Grant-in-Aid for Scientific Research (C), 25330353.

\section{References}

1. Euler T, Haverkamp S, Schubert T, Baden T. Retinal bipolar cells: elementary building block of vision. Nat Rev Neurosci. 2014;15:507-519.

2. DeVries SH. Bipolar cells use kainate and AMPA receptors to filter visual information into separate channel. Neuron. 2000;28:847-856.

3. Lindstrom SH, Ryan DG, Shi J, DeVries SH. Kainate receptor subunit diversity underlying response diversity in retinal Off bipolar cells. J Physiol. 2014;592:1457-1477.

4. Ichinose T, Hellmer CB. Differential signalling and glutamate receptor compositions in the Off bipolar cell types in the mouse retina. J Physiol. 2016;596:883-894.

5. DeVries SH, Li W, Sazik S. Parallel processing in two transmitter microenvironments at the cone photoreceptor synapse. Neuron. 2006;50:735-748.

6. Nielson TA, DiGregorio DA, Silver RA. Modulation of glutamate mobility reveals the mechanism underlying slow-rising AMPAR EPSCs and the diffusion coefficient in the synaptic cleft. Neuron. 2004;42:757-771.

7. Pan Z, Hu H. Voltage-dependent Na+ current in mammalian retinal cone bipolar cells. J Neurophysiol. 2000;84:2564-2571.

8. $\mathrm{Hu} \mathrm{H}, \mathrm{Pan} \mathrm{Z}$. Differential expression of $\mathrm{K}+$ currents in mammalian retinal bipolar cells. Vis Neurosci. 2002;19:163-173.

9. Ma Y, Cui J, Hu H, Pan Z. Mammalian retinal bipolar cells express inwardly rectifying K+ currents (Ikir) with a different distribution than that of Ih. J Neurophysiol. 2003;90:3479-3489. 
10. Usui S, Ishihara A, Kamiyama Y. Ionic current model of bipolar cells in the lower vertebrate retina. Vis Neurosci.1996;36:4069-4076.

11. Ishihara A. Simulation analysis of temporal coding by ionotropic glutamate receptors of retinal Off-type bipolar cells. Sensor and Materials 2018;30:299-313.

12. van Hateren JH, Snippe HP. Simulating human cones from mid-mesopic up to high-photopic luminances. J Vis. 2007;7:1-11.

13. Ishihara A, Kamiyama Y, Usui S. Analysis of light response of the retinal bipolar cells based on ionic current model. Computational Neuroscience: Trend in Research. New York: Plenum Press, 1998; 203-209.

14. Ishihara A. Simulation study of information transmission in OFF cone bipolar cell pathway. ARVO Annual Meeting. Honolulu, HW, 2018; Abstract 1873-C0177.

15. Ma Y, Cui J, Pan Z. Heterogeneous expression of voltage-dependent $\mathrm{Na}+$ and $\mathrm{K}+$ channel in mammalian retinal bipolar cells. Vis Neurosci. 2005;22:119-133.

16. Ichinose T, Shields CR, Lukasiewicz PD., Sodium channels in transient retinal bipolar cells enhance visual responses in ganglion cells. J Neurosci. 2005;25:1856-1865. 